\title{
A Hybrid Fusion Algorithm for Integrated INS/UWB Navigation and Its Application in Vehicle Platoon Formation Control
}

\author{
Yingmao $\mathrm{Lu}^{1}$, Jianjun $\mathrm{Yi}^{1, *}$, Liang $\mathrm{He}^{2,3}$, Xiaomin $\mathrm{Zhu}^{1}$ and Pengfei $\mathrm{Liu}^{2,3}$ \\ ${ }^{1}$ Department of Mechanical Engineering, East China University of Science and Technology, Shanghai 200237, China \\ ${ }^{2}$ Shanghai Aerospace Control Technology Institute, Shanghai 201109, China \\ ${ }^{3}$ Shanghai Key Laboratory of Aerospace Intelligent Control Technology, Shanghai 201109, China \\ ${ }^{*}$ Corresponding author
}

\begin{abstract}
The vehicle positioning technology is playing an important role in Cooperative Vehicle Infrastructure System (CVIS). In those single positioning modes, the precision of wireless positioning is greatly affected by the environment. However, the accumulative errors of inertial navigation system (INS) and positioning can not be eliminated. In this paper, a hybrid fusion algorithm based on UWB (Ultra Wide Band) and INS is proposed and applied to the vehicle platoon formation control system. The hybrid fusion algorithm can overcome the shortcomings of non-line-of-sight (NLOS) problem in UWB positioning algorithm and accumulative errors of INS. This algorithm can also significantly enhance positioning accuracy in vehicle platoon formation control. The application scene consists of three types: road scene, junction scene and parking lot scene. The hybrid fusion algorithm can be used in combination with different scenarios to ensure the smooth operation of the vehicle platoon.
\end{abstract}

Keywords-UWB (Ultra Wide Band); INS (inertial navigation system); hybrid fusion algorithm; vehicle platoon formation control

\section{INTRODUCTION}

Vehicle location technology is the key to Cooperative Vehicle Infrastructure System (CVIS). The vehicle positioning technology mainly uses Dead Reckoning (DR), subgrade positioning and Global Navigation Satellite System (GNSS).

DR can maintain high accuracy in a short time, but will bring an accumulative error [1]. The main advantage of subgrade positioning technology is its efficiency and reliability. But it also can be affected by the environment dramatically. Especially when non-line-of-sight (NLOS), multipath or other circumstances exist. In these cases, the subgrade positioning error is huge ${ }^{[2]}$. GNSS has all-weather service, high efficiency and other functional advantages. Due to the multipath effect, the current GNSS positioning error is huge and significantly influenced by environmental factors. GNSS can not meet the requirements of positioning accuracy in CVIS ${ }^{[3]}$.

As each single means of positioning has its own inevitable shortcomings which can not be overcome. In practical applications, we usually use the combination of these positioning methods, which also combined with Map Matching (MM) method to improve the positioning accuracy. Integrated positioning algorithms such as GPS/MM, GPS/DR, GPS/DR/MM are wildly used. ${ }^{[4]}$

The vehicle platoon formation control is mainly divided into longitudinal control, lateral control and integrated control. Longitudinal control mainly includes adaptive cruise control system, obstacle avoidance system and autonomous vehicle platoon formation control system. Lateral control includes lane change warning system, lane keeping system, vehicle tracking system and so on. Integrated control is a combination of longitudinal and lateral control. In vehicle platoon formation control, there is a very high demand on the vehicle's distance in the vehicle platoon formation, which includes the front-to-back and left-right distances. So it requires higher positioning accuracy.

In this paper, the hybrid fusion algorithm is a combination of UWB positioning and INS technology which is a new form of the above multiple combined positioning methods. The hybrid fusion algorithm combines the advantages of overall high accuracy in UWB positioning and high accuracy within a short time in INS positioning. It can supply a high-precision positioning and communication, collision warning and so on in the vehicle platoon formation control. This is a new exploration and new application of this combination of positioning methods ${ }^{[5 \sim 8]}$.

The UWB positioning technology, UWB module selection, embedded hardware and software design and system integration program are introduced in first section. Then, the UWB and INS data fusion localization algorithm based on Federated Kalman Filter is proposed. And the algorithm is applied to vehicle distance estimation and collision prewarning algorithm in vehicle platoon formation control. Finally, this paper analyzes the application of hybrid fusion algorithm in different scenarios of vehicle platoon formation control.

\section{Overall Program Design}

\section{A. Overview of the UWB Positioning Technology}

UWB (Ultra Wide Band) is a carrierless communication technology that uses nanoseconds to microseconds nonsinusoidal narrow pulses to transmit data (Figure 1). UWB has other incomparable communication system features include: 
High rate and large channel capacity; Suitable for short distance communication; Good coexistence and confidentiality; Strong multipath resolution and high positioning accuracy; Small size, low power consumption; etc ${ }^{[9]}$. UWB was used in the near-field high-speed data transmission early. In recent years began to use its sub-nanosecond ultra-narrow pulse in close-range accurate positioning. Precise Positioning is an applications of UWB in the field of IEEE802.15.4a. It is a lowspeed low-power transmission for precise positioning of the technology. The rise of UWB technology has a great promotive effect for the WSN positioning. Compared with other WSN positioning methods, the positioning accuracy of UWB technology has been greatly improved, and the cost is much less than expensive positioning methods such as laser positioning. UWB has the advantages of low cost, antimultipath interference and penetrating ability in positioning. So it can be applied to the positioning and tracking of stationary or moving objects and personnel, and can provide very accurate positioning accuracy. Currently UWB technology is mainly used in indoor positioning, less application in vehicle positioning. The United States PAL650 system using UWB system successfully solved the problem of container transport vehicles positioning. Ubisense UK's UWB positioning system has been able to complete the precise positioning of $20 \sim 50 \mathrm{~m}$ range of $15 \mathrm{~cm}$, so that it can be applied in the parking lot, logistics and other vehicle related aspects.

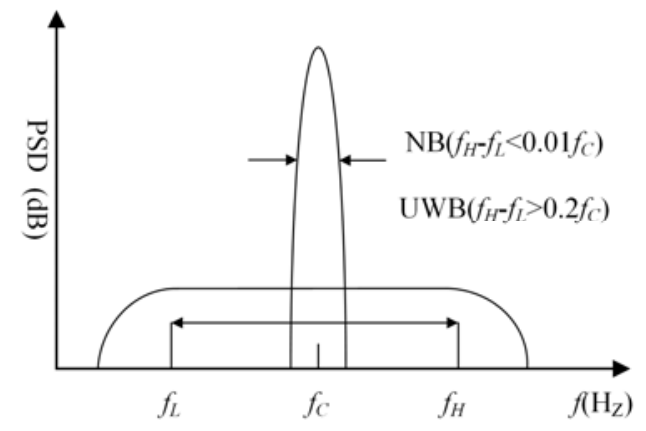

FIGURE I. SIGNAL IN ACCORDANCE WITH THE BANDWIDTH CLASSIFICATION

\section{B. UWB Selection, Hardware Design, Integration and Embedded Software Development}

In this paper, the overall scheme of UWB vehicle positioning is shown in Figure 2, in which each vehicle is equipped with UWB positioning nodes. And it is arranged with fixed UWB anchor nodes in the whole space. The positioning node sends data to the anchor node according to a certain frequency. After receiving the data, the anchor node gives a response and calculates the ranging.

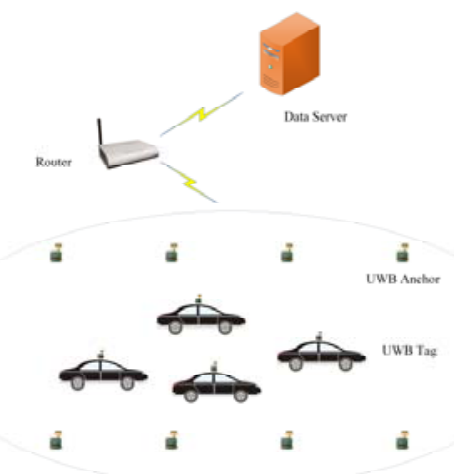

FIGURE II. SYSTEM OVERALL PROGRAM

After receiving the signal and calculating the distance value with each positioning node, the UWB anchor node sends the positioning result information to the dedicated router through the TCP/IP communication module. And the dedicated router collects information and sends it to the data center uniformly through the network. The high-performance computer in the

data center performs the uniform processing on the ranging data of each UWB node and quickly locates each UWB node.

UWB positioning nodes fall into two categories, of which the onboard control system serves as a mobile node or a label node, and which is fixed in an indoor environment as a reference node or anchor node. Anchor nodes and tag nodes can have the same hardware design. Different positioning roles can be performed by changing the configuration on the software.

UWB transceiver is the core of UWB communication and positioning among the positioning nodes. Take DecaWave DW1000 for example. The UWB transceiver chip can communicate with the host through the SPI interface. Its internal structure is shown in Figure 3.At the same time the UWB transceiver chip has the following excellent features: Supports the highest $6.8 \mathrm{Mbps}$ communication rate; Farthest communication distance up to 290m under 110kbps; High antimultipath ability; Excellent NLOS communication ability; Low-power control strategy.

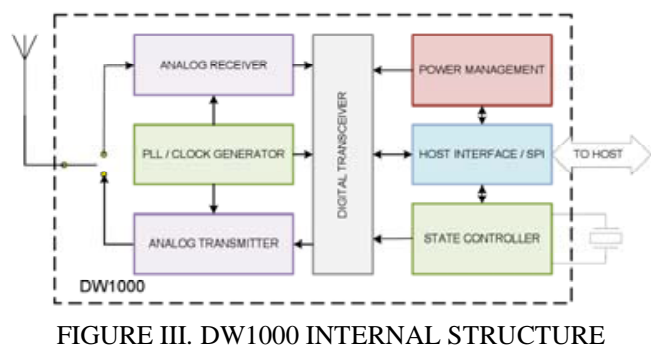

In addition to transceiver chip, UWB positioning node also need a host to configure its state and transmit data. In this paper, a STM32F105RCT6 chip is used as a controller to communicate with the UWB transceiver through the SPI interface. At the same time, the host chip communicates with other hosts through TCP / IP or USB interface and transmits the ranging data to other nodes. The main structure of UWB positioning node shown in Figure 4. 


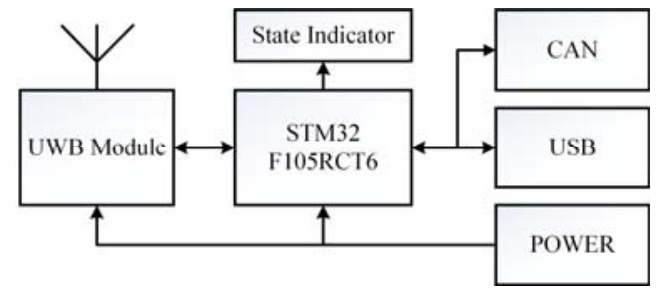

FIGURE IV. HARDWARE STRUCTURE OF UWB NODE

In this paper, the host controller of vehicle which needs to be integrated with the UWB as tag node connects to the UWB node via CAN. And the vehicle's controller obtain the information such as the wireless connection status and the ranging result from the UWB node. At the same time, the vehicle controller supplies power to the UWB node through the power interface. In combination with the software design and switch module, the node can be used as the label or anchor node, which greatly increases the universality of the UWB node.

In this paper as a dedicated router for information transfer, the hardware structure is shown in Figure 5. The MCU module is developed using TI's Cortex-A8-based AM335X core chip. The chip has a wealth of industrial interfaces and is able to run Linux operating system, which provide a reliable guarantee to the development of hardware and software for the router. Dedicated router includes a power module. When the power module can not supply power, the backup power will be enabled to ensure the reliable operation of the system. The LCD module provides the working status display of the current router, such as the information of the number of each UWB node currently connected, the information of the death node, the connection status of the node, and so on.

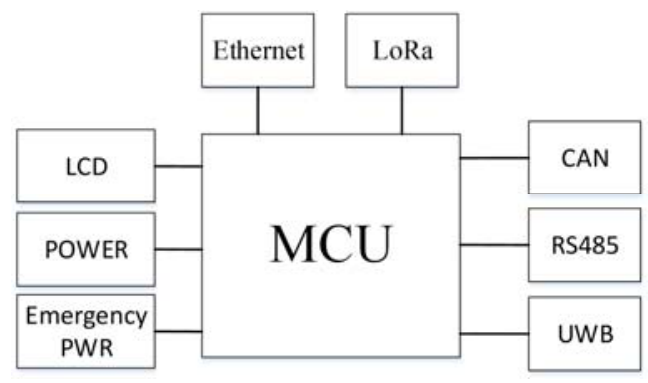

FIGURE V. HARDWARE STRUCTURE OF ROUTER

As a dedicated router, it has a wealth of peripheral interfaces. First of all, it has UWB module to communicate with other UWB nodes in the system. RS485 and CAN are the mainstream industrial application interfaces that ensure reliable data communication between this dedicated router and other nodes. The Ethernet interface is a communication interface for uploading UWB positioning data. When the router is in an environment that does not have reliable Ethernet access, data is communicated over an extra LoRa wireless network. As a longdistance wireless communication technology, LoRa can guarantee reliable data communication within $5 \mathrm{Km}$. Therefore, LoRa can greatly expand the use range of the router and reduce its dependence on the Internet.

\section{UWB Localization Algorithm and Its Data Fusion Algorithm with Inertial Positioning}

The basis of UWB positioning is to model the current environment effectively to express its environmental characteristics. UWB positioning is also a relative positioning method in the map model. So map creation is an important step in UWB positioning. There are two main ways of modeling the environment of the map: net/graph modeling method and gridbased modeling method. The former mainly includes modeling methods such as free space method and visual method. The latter grid-based modeling method is the grid map method. Because grid map modeling method is relatively simple to implement, and can be well applied to the modeling of the rule environment. Therefore, this paper intends to use the modeling method of grid map to model the vehicle operating environment. Although grid maps are easy to implement, the size of the grid directly affects the efficiency of path planning. Therefore, how to divide the environment, the precision of the grid needs to be further studied according to the specific environment so that the vehicle can achieve a better balance between the positioning accuracy and the efficiency, so as to meet the optimal posture determination of the vehicle under dynamic conditions. At the same time, in grid maps, the expression of irregular obstacles is also an important aspect that affects their accuracy and efficiency ${ }^{[10,11]}$.

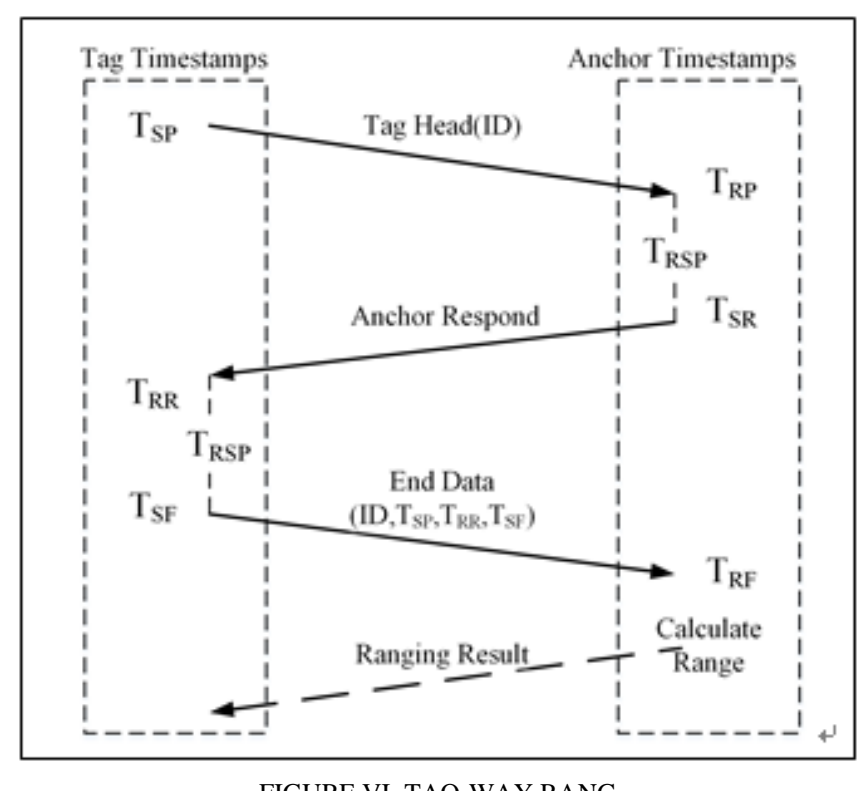

FIGURE VI. TAO-WAY RANG

In order to solve the wireless positioning of the time synchronization problem, this paper uses a Two-Way Rang (TWR) method based on time of flight. The one-way flight time is calculated by passing the signal's timestamps when two nodes communicate bi-directionally, thereby being immune to clock synchronization. The principle of TWR method is shown in Figure 6. In Figure 6, the left side represents the label node and the right side is the anchor node. The tag node starts the ranging process at a certain time interval. The anchor node will always keep listening state and respond to the ranging signal and calculate the distance between nodes. A complete ranging process is as follows: 
For tag nodes, the signal transmission delay $\mathrm{T}_{\mathrm{TRT}}$ can be used $\left(\mathrm{T}_{\mathrm{RR}}-\mathrm{T}_{\mathrm{SP}}\right)$ to represent. For anchor nodes, the round trip delay $\mathrm{T}_{\mathrm{ART}}$ can be expressed as $\left(\mathrm{T}_{\mathrm{RF}}-\mathrm{T}_{\mathrm{SR}}\right)$. Obviously in addition to the flight time of the signal round trip delay time, there is a response time from the received transmission of a node. Therefore, removing the response time $\left(T_{R S P}=T_{S R}-T_{R P}\right)$ of the anchor node from TTRT and removing the response time $\left(T_{R S P}=T_{S F}-T_{R R}\right)$ of the label node from T_ART can obtain the net time for the signal to travel back and forth between two nodes. In order to eliminate the impact caused by the clock discrepancy of the terminal equipment on both sides, the time of the round trip on both ends is averaged, and then divided by 2 to obtain the one-way time. That is, the one-way flight time can be calculated by equation (1):

$$
\mathrm{TOF}=\left(\left(\mathrm{T}_{\mathrm{RR}}-\mathrm{T}_{\mathrm{SP}}\right)-\left(\mathrm{T}_{\mathrm{SR}}-\mathrm{T}_{\mathrm{RP}}\right)+\left(\mathrm{T}_{\mathrm{RF}}-\mathrm{T}_{\mathrm{SR}}\right)-\left(\mathrm{T}_{\mathrm{SF}}-\mathrm{T}_{\mathrm{RR}}\right)\right) / 4
$$

Multiplying the calculated flight time TOF by the transmission speed of the radio waves (the speed of light c), we obtain the distance $\mathrm{L}$ between the two nodes. Through the above method, the requirement of extremely high time synchronization in UWB positioning can be avoided, and the difficulty and cost of implementation are greatly reduced.

Based on the UWB ranging above, accurate positioning can be achieved by ranging projection and trilateral positioning algorithm. Based on the UWB positioning data and the INS data of the vehicle, the data fusion of the two positioning algorithms is realized. In this paper, the Federated Kalman Filter is used to fuse the above two positioning methods.

Kalman filter is a class of optimal filters for Gaussian noise in linear systems. And it is a mainstream technology in the field of information fusion. Federated Kalman filter is the most widely used distributed Kalman filter. It is designed into different structures according to system requirements. And it can distributes system information among subsystems through the principle of information distribution.

In federated Kalman filter-based fusion localization, UWB positioning and inertial positioning are used as subsystems. The structure of it is shown in Figure 7. Firstly, each subsystem inputs data $\mathrm{Zi}$ to a respective local filter. Each local filter predicts and updates the data. Then input the local estimated value $\mathrm{Xi}$ and the covariance matrix $\mathrm{Pi}$ to the main filter. The main filter do the time update and data fusion to obtain the global optimum estimate. After that the main filter returns the global estimate $\mathrm{Xg}$ and the covariance matrix Pg to the subfilter through the information distribution principle, and resets the estimated value of the sub-filter and the covariance matrix. Then the main filter can output the optimal positioning estimation.

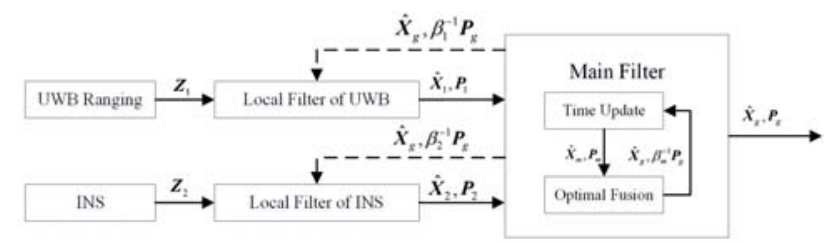

FIGURE VII. HYBRID FUSION ALGORITHM BASED ON FEDERATED KALMAN FILTER

\section{Vehicle Distance Estimation and Collision Warning Algorithm in Vehicle Platoon Formation Control}

In addition to the vehicle location, the system also need to obtain the current pose information of the vehicle, speed information, and so on. When the vehicle get into the positioning area, the collision warning system is to work. Vehicle on-board unit acquires vehicle status information such as vehicle speed, position, acceleration, angular velocity and course angle through UWB and INS. Then send these information to the router so that to upload to the server via UWB anchor node. The server performs data processing of the vehicle collision algorithm to detect whether the vehicle collision exists. If there is no vehicle collision, the vehicle can safely pass the area in its current state. If there is a vehicle collision, the severity of the collision is evaluated to calculate the safety interval of the vehicle speed. And according to the collision of different security levels to the collision may be sent to vehicles warning information. Early warning information is transmitted to the vehicle control unit via UWB wireless communications. The vehicle will alert the driver take the necessary measures to avoid the impending collision of vehicles.

\section{E. Application of Hybrid Fusion Algorithm in Vehicle Platoon Formation Control}

This application scene of the hybrid fusion algorithm in the Vehicle platoon formation control is divided into three types: roads, junctions and parking. Described below separately:

1) Roads Scene: In the road scene(Figure 8), the vehicles in the vehicle platoon formation realize the networking through UWB communication, and make use of the hybrid fusion algorithm of UWB and INS to locate the relative position. Based on this, the vehicle distance is estimated, and the vehicle distance information is sent to the moving vehicles in real time. Then the system realized the precise control of the distance of the vehicle platoon formation and the complete vehicle platoon formation early warning system.

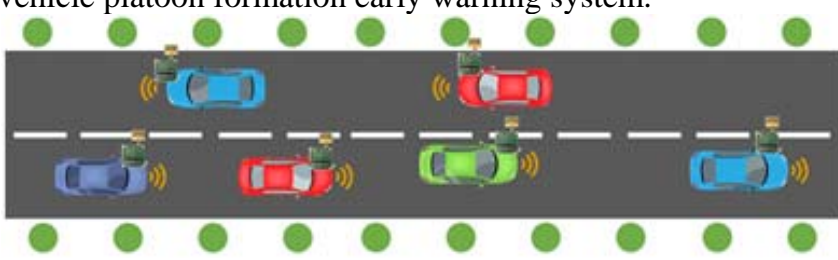

FIGURE VIII. ROADS SCENE APPLICATION OF HYBRID FUSION ALGORITHM

2) Junctions: In the intersection scene, the vehicles in the vehicle platoon formation communicate with the UWB anchor nodes at the intersection. The hybrid fusion algorithm can calculate the real-time position and driving direction of the vehicle and combine with the electronic map to achieve collision warning and lane keeping in the vehicle platoon formation. Lane change warning function. 


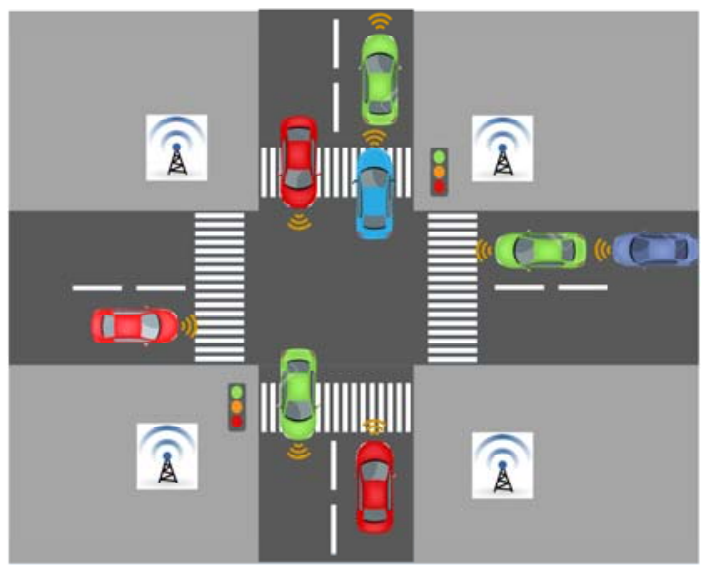

FIGURE IX. JUNCTIONS SCENE APPLICATION OF HYBRID FUSION ALGORITHM

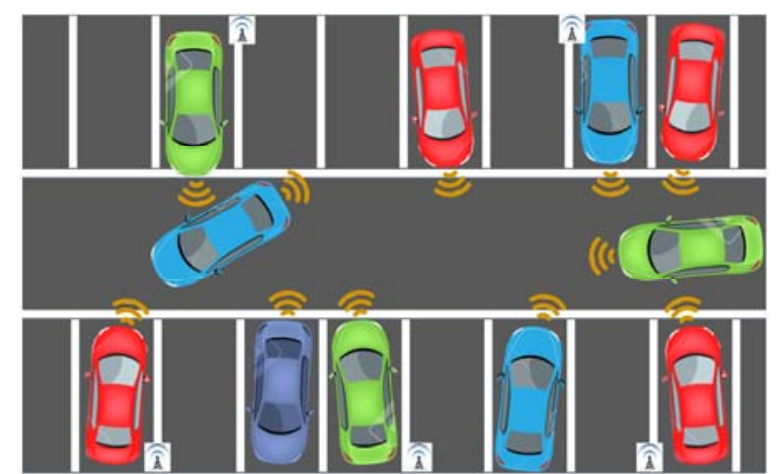

FIGURE X. PARKING SCENE APPLICATION OF HYBRID FUSION ALGORITHM

3) Parking: In the parking lot scene(Figure 10), vehicles in the vehicle platoon formation communicate with UWB anchor nodes, and the hybrid fusion algorithm can be used to calculate the real-time location and area information of the vehicle. In combination with the electronic map, the vehicle distance prediction in the parking lot (including the longitudinal and horizontal car), to ensure that the vehicle entering the collision prevention process early warning.

\section{CONCLUSION}

In this paper, a completed vehicle platoon formation control system is proposed which mainly contains a hybrid fusion algorithm for integrated INS/UWB navigation and its application in this system. Frist of all, the INS and UWB

positioning data is collected, including final location data and intermediate data. The TWR (Two-Way Range) method based on time of flight can solve the time synchronization problem in UWB positioning algorithm. Moreover, the hybrid fusion algorithm uses Federated Kalman Filter integrate INS/UWB positioning data to get an accuracy location. The UWB model selection, location scheme confirmation, hardware and software design, and so on are also put forward among them in this paper. At last, the hybrid fusion application in vehicle platoon formation control is analyzed which includes vehicle distance estimation, collision warning algorithm and vehicle platoon formation control in different scenarios.

\section{ACKNOWLEDGMENT}

This paper was supported by the Research Foundation of Science and Technology Commission of Shanghai under Grant No. 10DZ1500200, the Natural Science Fund of China (NSFC) under Grant Nos. 51575186, 51275173, and 50975088, the Fundamental Research Funds for the Central Universities under Grant No. WH0913009, Shanghai Pujiang Program under Grant No. PJ201000353, and Shanghai Software and IC industry Development Special Fund under Grant No. 120493.

\section{REFERENCES}

[1] Hye Ri Park, Dong Jun Hyun, Hyun Seok Yang, et al. A Dead Reckoning Sensor System and a Tracking Algorithm for Mobile Robot. Japan: ICROS-SICE International Joint Conference, 2009.

[2] Tim, Tau, Hsieh. Using sensor networks for highway and traffic application. IEEE Potentials Apprial, 2004, (24): 13-16.

[3] Vicent Pereira, Audrey Giremus, and Eric Grivel. Modeling of Multipath Environment Using Copuals for Partical Filtering Based GPS Navigation. IEEE Signal Processing Letters. 2012, 19(6): 360-363.

[4] Seong Yun Cho, Wan Sik Choi. Robust Positioning Technique in Lowcost DR/GPS for Land Navigation. IEEE Transaction on Instrumentation and Measurement. 2006, 55(4): 1132-1142.

[5] Ghasemzadeh H, Behrangi E, Azgomi M A. Conflict-free scheduling and routing of automated guided vehicles in mesh topologies. Robotics \& Autonomous Systems, 2009, 57(6): 738-748.

[6] Qigao Fan, Yaheng Wu, Jing Hui, Lei Wu, Zhenzhong Yu, Lijuan Zhou, Chingiz Hajiyev. Integrated Navigation Fusion Strategy of INS/UWB for Indoor Carrier Attitude Angle and Position Synchronous Tracking. The Scientific World Journal, 2014(2014).

[7] KOK M, HOL J D, SCHON $\mathrm{T}$ B. Indoor positioning using ultrawideband and inertial measurements. IEEE Transactions on Vehicular Technology, 2015, 64(4):1293-1303.

[8] Accurate pedestrian indoor navigation by tightly coupling foot-mounted IMU and RFID measurements. IEEE Transactions on Instrumentation and Measurement, 2012,61(1):178-188.

[9] FCC 02-48, FIRST REPORT AND ORDER. Adopted: 2002, February 14, Released: 2002.

[10] Cui Bing-bo, Chen Xi-yuan, Xu Yuan, et al. Performance analysis of improved iterated cubature Kalman filter fand its application to GNSS/INS. ISA Transactions, 2017, 66:460-468.

[11] Xu Yuan, Chen Xi-yuan, Wang Yi-min, et al. Improving indoor pedestrian navigation method using low cost foot-mounted AHRS and shoulder-mounted compass. Journal of Chinese Inertial Technology, 2016, 24(3):325-329. 